\title{
Impulsive Stabilization for Control and Synchronization of Chaotic Systems: Theory and Application to Secure Communication
}

\author{
Tao Yang, Member, IEEE, and Leon O. Chua, Fellow, IEEE
}

\begin{abstract}
Impulsive control of a chaotic system is ideal for designing digital control schemes where the control laws are generated by digital devices which are discrete in time. In this paper, several theorems on the stability of impulsive control systems are presented. These theorems are then used to find the conditions under which the chaotic systems can be asymptotically controlled to the origin by using impulsive control. Given the parameters of the chaotic system and the impulsive control law, an estimation of the upper bound of the impulse interval is given. We also present a theory of impulsive synchronization of two chaotic systems. A promising application of impulsive synchronization of chaotic systems to a secure communication scheme is presented. In this secure communication scheme, the transmitted signals are divided into small time frames. In each time frame, the synchronization impulses and the scrambled message signal are embedded. Conventional cryptographic methods are used to scramble the message signal. Simulation results based on a typical chaotic system; namely, Chua's oscillator, are provided.
\end{abstract}

Index Terms - Chaotic secure communication, Chua's oscillator, continuous cryptographic function, impulsive control, impulsive synchronization.

\section{INTRODUCTION}

$\mathbf{S}$ INCE THE seminal paper of Ott, Grebogi, and Yorke (OGY) [2], several methods for control and stabilization of chaotic motions have recently been presented [3]-[6]. In view of the rich dynamics of chaotic systems, there exists a large variety of approaches for controlling such systems. Some of these approaches include adaptive control [4], [5], error-feedback control [7], time-delay feedback control [7], OGY method [2], predictive Poincaré control [8], occasional proportional feedback control [9], and impulsive control [6], [15]-[20].

In fact, the predictive Poincaré control and the occasional proportional feedback control are two impulsive control schemes with varying impulse intervals. Impulsive control is attractive because it allows the stabilization of a chaotic system using only small control impulses, and it offers a direct

Manuscript received January 30, 1997; revised June 16, 1997. This work was supported by the Office of Naval Research under Grant N00014-96-10753. This paper was recommended by Guest Editor M. J. Ogorzałek.

The authors are with the Electronics Research Laboratory, Department of Electrical Engineering and Computer Sciences, University of California, Berkeley, CA 94720 USA.

Publisher Item Identifier S 1057-7122(97)07321-2. method for modulating digital information onto a chaotic carrier signal for spread spectrum applications. However, due to a lack of effective tools for analyzing impulsive differential equations [1], most impulse control schemes had been designed mainly by trial-and-error. The study of the stability of an impulsive differential equation is much more difficult than that of its "corresponding" differential equation [10]. For example, consider the impulsive system

$$
\left\{\begin{array}{l}
\dot{\mathrm{x}}=A \mathrm{x}, \\
\left.\Delta \mathrm{x}\right|_{t=\tau_{i}}=B \mathrm{x}
\end{array} \quad t \neq \tau_{i}\right.
$$

where $A$ and $B$ are two constant matrices, and $\left.\Delta \mathbf{x}\right|_{t=\tau_{i}} \triangleq \mathbf{x}\left(\tau_{i}^{+}\right)-\mathbf{x}\left(\tau_{i}^{-}\right), \mathbf{x}\left(\tau_{i}^{-}\right)$, and $\mathbf{x}\left(\tau_{i}^{+}\right)$being the left and right limit of $\mathbf{x}(t)$ at $t=\tau_{i}$. The solution of the above system is given by

$$
\mathrm{x}\left(t, \mathrm{x}_{0}\right)=\mathbf{X}\left(t, \mathrm{x}_{0}\right) \mathrm{x}_{0}
$$

where

$$
\begin{gathered}
\mathbf{X}\left(t, \mathbf{x}_{0}\right)=e^{A\left(t-\tau_{i}\right)} \prod_{\substack{t_{0}<\tau_{j}<t \\
\tau_{0}}=t_{0}, \quad \tau_{i}<t \leq \tau_{i+1} .}(I+B) e^{A\left(\tau_{j}-\tau_{j-1}\right)}
\end{gathered}
$$

As can be seen from this formula, it is not possible in the general case to give necessary and sufficient conditions for stability of solutions of the above system in terms of the eigenvalues of the matrix of this system, which is possible for systems of ordinary differential equations with constant coefficients.

In this paper, we investigate the stability of impulsively controlled chaotic systems. First, the stability of the trivial solution of a kind of impulsive differential equation is studied. Then the theoretical results are used to study the conditions under which an impulsive control of Chua's oscillator is asymptotically stable. An estimate of the upper bound of the impulsive interval is also presented.

Then, an impulsive control theory is used to study the impulsive synchronization of two chaotic systems. We first show that the impulsive synchronization problem is an impulsive control problem. Then a theorem is given for guaranteeing the asymptotic stability of impulsive synchronization. Since only the synchronization impulses are sent to the driven system in 


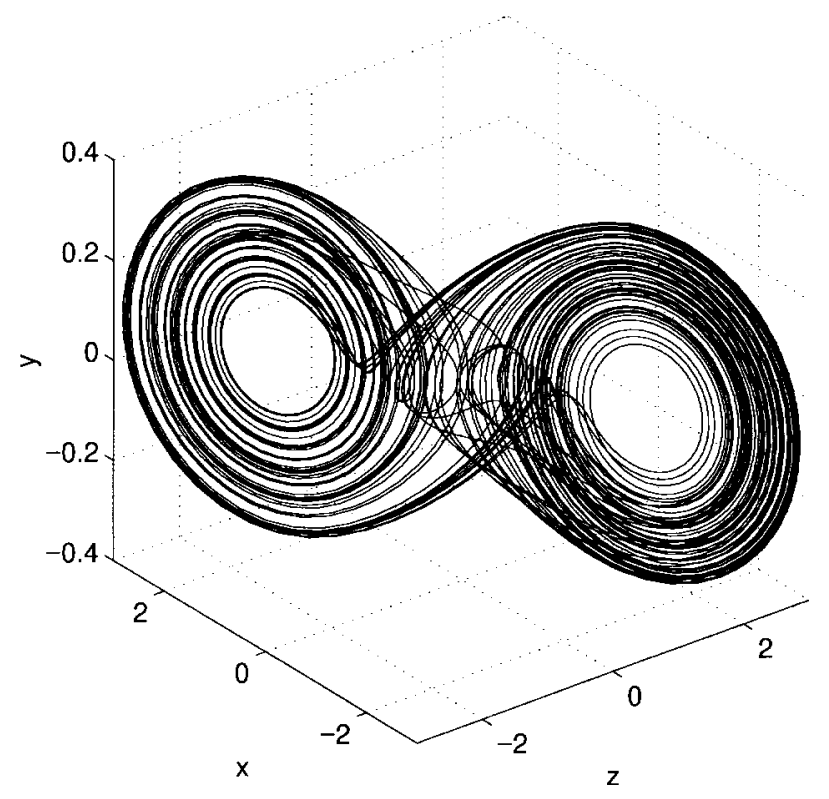

Fig. 1. The Chua's double scroll attractor.

an impulsive synchronization scheme, the information redundancy in the transmitted signal is reduced. In this sense, even low-dimensional chaotic systems can provide high security. In this paper, we will use impulsive synchronization to develop a new framework for chaotic secure communication.

The organization of this paper is as follows. In Section II, a theory on the stability of impulsive differential equations is given. In Section III, a stability criterion for impulsive control of Chua's oscillator is presented. In Section IV, simulation results on the impulsive control of Chua's oscillator are provided. In Section V, the theory and simulation results of impulsive synchronization of Chua's oscillators are presented. In Section VI, application of impulsive synchronization to secure communication is presented. In Section VII, some concluding remarks are given.

\section{BASIC ThEORY OF IMPULSIVE DIFFERENTIAL EQUATIONS}

Consider the general nonlinear system

$$
\dot{\mathrm{x}}=\mathbf{f}(t, \mathbf{x})
$$

where $\mathbf{f}: \mathbf{R}_{+} \times \mathbf{R}^{n} \mapsto \mathbf{R}^{n}$ is continuous, $\mathbf{x} \in \mathbf{R}^{n}$ is the state variable, and

$$
\dot{\mathbf{x}} \triangleq \frac{d \mathbf{x}}{d t} .
$$

Consider a discrete set $\left\{\tau_{i}\right\}$ of time instants, where

$$
\begin{aligned}
0<\tau_{1} & <\tau_{2}<\cdots<\tau_{i}<\tau_{i+1}<\cdots, \\
\tau_{i} & \rightarrow \infty \text { as } i \rightarrow \infty .
\end{aligned}
$$

Let

$$
U(i, \mathbf{x})=\left.\Delta \mathbf{x}\right|_{t=\tau_{i}} \triangleq \mathbf{x}\left(\tau_{i}^{+}\right)-\mathbf{x}\left(\tau_{i}^{-}\right)
$$

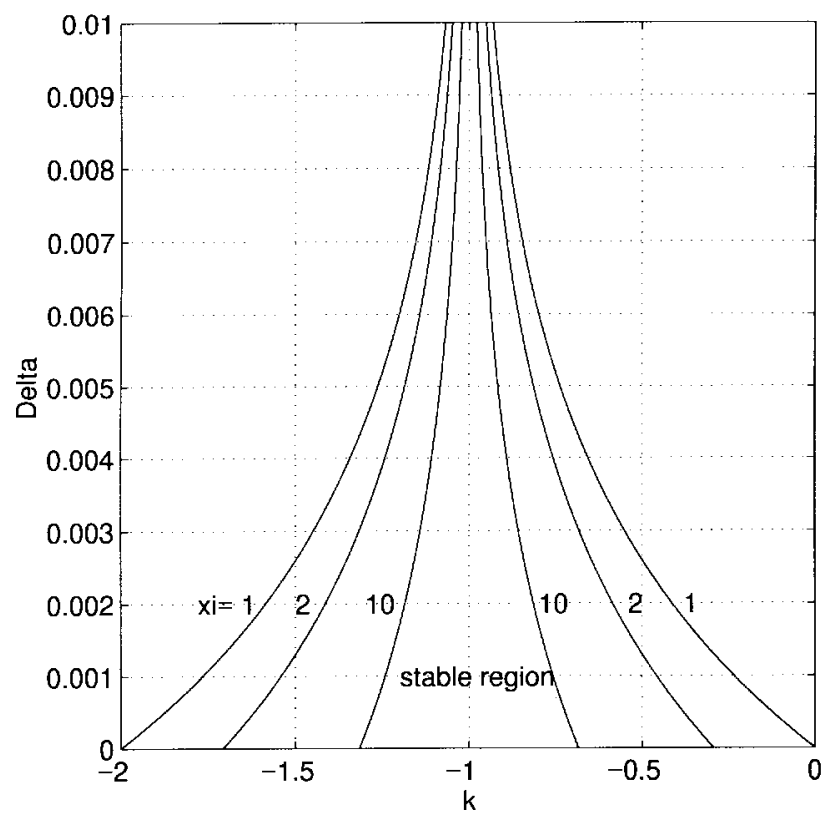

Fig. 2. Estimate of the boundaries of stable regions with different $\xi$ 's used in simulation 1.

be the "jump" in the state variable at the time instant $\tau_{i}$. Then this impulsive system is described by

$$
\left\{\begin{array}{ll}
\dot{\mathbf{x}}=\mathbf{f}(t, \mathbf{x}), & t \neq \tau_{i} \\
\Delta \mathbf{x}=U(i, \mathbf{x}), & t=\tau_{i} \\
\mathbf{x}\left(t_{0}^{+}\right)=\mathbf{x}_{0}, & t_{0} \geq 0, i=1,2, \cdots
\end{array} .\right.
$$

This is called an impulsive differential equation [1]. To study the stability of the impulsive differential equation (6) we use the following definitions and theorems [1].

Definition 1: Let $V: \mathbf{R}_{+} \times \mathbf{R}^{n} \mapsto \mathbf{R}_{+}$, then $V$ is said to belong to class $\mathcal{V}_{0}$ if

1) $V$ is continuous in $\left(\tau_{i-1}, \tau_{i}\right] \times \mathbf{R}^{n}$ and for each $\mathbf{x} \in \mathbf{R}^{n}$, $i=1,2, \cdots$,

$$
\lim _{(t, \mathbf{y}) \rightarrow\left(\tau_{i}^{+}, \mathbf{x}\right)} V(t, \mathbf{y})=V\left(\tau_{i}^{+}, \mathbf{x}\right)
$$

exists;

2) $V$ is locally Lipschitzian in $\mathbf{x}$.

Definition 2: For $(t, \mathbf{x}) \in\left(\tau_{i-1}, \tau_{i}\right] \times \mathbf{R}^{n}$, we define

$$
\begin{aligned}
D^{+} V(t, \mathbf{x}) \triangleq & \lim _{h \rightarrow 0} \sup \frac{1}{h}\{V[t+h, \mathbf{x}+h \mathbf{f}(t, \mathbf{x})] \\
& -V(t, \mathbf{x})\} .
\end{aligned}
$$

Definition 3. Comparison System: Let $V \in \mathcal{V}_{0}$ and assume that

$$
\begin{cases}D^{+} V(t, \mathbf{x}) \leq g[t, V(t, \mathbf{x})], & t \neq \tau_{i} \\ V[t, \mathbf{x}+U(i, \mathbf{x})] \leq \psi_{i}[V(t, \mathbf{x})], & t=\tau_{i}\end{cases}
$$

where $g: \mathbf{R}_{+} \times \mathbf{R}_{+} \mapsto \mathbf{R}$ is continuous and $\psi_{i}: \mathbf{R}_{+} \mapsto \mathbf{R}_{+}$ is nondecreasing. Then the system

$$
\left\{\begin{array}{l}
\dot{w}=g(t, w), \quad t \neq \tau_{i} \\
w\left(\tau_{i}^{+}\right)=\psi_{i}\left[w\left(\tau_{i}\right)\right] \\
w\left(t_{0}^{+}\right)=w_{0} \geq 0
\end{array}\right.
$$

is called the comparison system of (6). 
Definition 4:

$$
S_{\rho}=\left\{\mathrm{x} \in \mathbf{R}^{n} \mid\|\mathrm{x}\|<\rho\right\}
$$

where $\|\cdot\|$ denotes the Euclidean norm on $\mathbf{R}^{n}$.

Definition 5: A function $\alpha$ is said to belong to class $\mathcal{K}$ if $\alpha \in C\left[R_{+}, R_{+}\right], \alpha(0)=0$, and $\alpha(x)$ is strictly increasing in $x$.

Assumptions: $\mathrm{f}(t, 0)=0, U(i, 0)=0$, and $g(t, 0)=0$ for all $i$.

Remark: With the above assumptions, we find that the trivial solutions of (6) and (10) are identical for all time except at the discrete set $\left\{\tau_{i}\right\}$.

Theorem 1. [1, Theorem 3.2.1, p. 139]: Assume that the following three conditions are satisfied.

1) $V: \mathbf{R}_{+} \times S_{\rho} \mapsto \mathbf{R}_{+}, \rho>0, V \in \mathcal{V}_{0}, D^{+} V(t, \mathbf{x}) \leq$ $g[t, V(t, \mathbf{x})], t \neq \tau_{i}$.

2) There exists a $\rho_{0}>0$ such that $\mathrm{x} \in S_{\rho_{0}}$ implies that $\mathrm{x}+U(i, \mathrm{x}) \in S_{\rho_{0}}$ for all $i$ and $V[t, \mathrm{x}+U(i, \mathbf{x})] \leq$ $\psi_{i}[V(t, \mathbf{x})], t=\tau_{i}, \mathbf{x} \in S_{\rho_{0}}$.

3) $\beta(\|\mathbf{x}\|) \leq V(t, \mathbf{x}) \leq \alpha(\|\mathbf{x}\|)$ on $\mathbf{R}_{+} \times S_{\rho}$, where $\alpha(\cdot), \beta(\cdot) \in \mathcal{K}$.

Then the stability properties of the trivial solution of the comparison system (10) imply the corresponding stability properties of the trivial solution of (6).

Theorem 2. [1, Corollary 3.2.1., p. 142]: Let $g(t, w)=$ $\dot{\lambda}(t) w, \lambda \in C^{1}\left[\mathbf{R}_{+}, \mathbf{R}_{+}\right], \psi_{i}(w)=d_{i} w, d_{i} \geq 0$ for all $i$. Then the origin of system (6) is asymptotically stable if the conditions

$$
\lambda\left(\tau_{i+1}\right)+\ln \left(\gamma d_{i}\right) \leq \lambda\left(\tau_{i}\right), \text { for all } i, \text { where } \gamma>1
$$
and

$$
\dot{\lambda}(t) \geq 0
$$

are satisfied.

\section{Stabilization of Chua's OSCILLATOR USING IMPULSIVE CONTROL}

In this section, we study the impulsive control of Chua's oscillators [11] by applying the theory presented in the previous section. The dimensionless form of a Chua's oscillator is given by [11]

$$
\left\{\begin{array}{l}
\dot{x}=\alpha[y-x-f(x)] \\
\dot{y}=x-y+z \\
\dot{z}=-\beta y-\gamma z
\end{array}\right.
$$

where $f(x)$ is the piecewise-linear characteristics of the Chua's diode, which is given by

$$
f(x)=b x+\frac{1}{2}(a-b)(|x+1|-|x-1|)
$$

where $a<b<0$ are two constants.

Let $\mathbf{x}^{T}=(x, y, z)$, then we can rewrite the Chua's oscillator equation into the form

$$
\dot{\mathrm{x}}=A \mathrm{x}+\Phi(\mathrm{x})
$$

where

$$
\begin{aligned}
A & =\left(\begin{array}{rrr}
-\alpha & \alpha & 0 \\
1 & -1 & 1 \\
0 & -\beta & -\gamma
\end{array}\right), \\
\Phi(\mathbf{x}) & =\left[\begin{array}{c}
-\alpha f(x) \\
0 \\
0
\end{array}\right] .
\end{aligned}
$$

The impulsive control of a Chua's oscillator is then given by

$$
\left\{\begin{array}{l}
\dot{\mathrm{x}}=A \mathrm{x}+\Phi(\mathrm{x}), \quad t \neq \tau_{i} \\
\left.\Delta \mathrm{x}\right|_{t=\tau_{i}}=B \mathrm{x}
\end{array} .\right.
$$

Since the system in (18) has the general form of a kind of nonlinear system, we can give a general result in following lemma.

Lemma 1: Let $n \times n$ matrix $\Gamma$ be symmetric and positive definite, and $\lambda_{1}>0, \lambda_{2}>0$ are, respectively, the smallest and the largest eigenvalues of $\Gamma$. Let

$$
Q=\Gamma A+A^{T} \Gamma
$$

where $A^{T}$ is the transpose of $A$, and $\lambda_{3}$ is the largest eigenvalue of $\Gamma^{-1} Q . \Phi(\mathbf{x})$ is continuous and $\|\Phi(\mathbf{x})\| \leq L\|\mathbf{x}\|$ where $L>0$ is a constant. $\lambda_{4}$ is the largest eigenvalue of the matrix

$$
\Gamma^{-1}\left(I+B^{T}\right) \Gamma(I+B) .
$$

$B$ is symmetric and the spectral radius of $I+B, \rho(I+$ $B) \leq 1$. Then the origin of impulsive control system (18) is asymptotically stable if

$$
\left(\lambda_{3}+2 L \sqrt{\frac{\lambda_{2}}{\lambda_{1}}}\right)\left(\tau_{k+1}-\tau_{k}\right) \leq-\ln \left(\gamma \lambda_{4}\right), \quad \gamma>1
$$

and

$$
\lambda_{3}+2 L \sqrt{\frac{\lambda_{2}}{\lambda_{1}}} \geq 0 .
$$

Proof: Construct a Lyapunov function $V(\mathbf{x})=\mathbf{x}^{T} \Gamma \mathbf{x}$, when $t \neq \tau_{k}$ we have

$$
\begin{aligned}
D^{+} V(\mathbf{x}) & =\mathbf{x}^{T}\left(A^{T} \Gamma+\Gamma A\right) \mathbf{x}+\left[\Phi^{T}(\mathbf{x}) \Gamma \mathbf{x}+\mathbf{x}^{T} \Gamma \Phi(\mathbf{x})\right] \\
& =\mathbf{x}^{T} Q \mathbf{x}+\left[\Phi^{T}(\mathbf{x}) \Gamma \mathbf{x}+\mathrm{x}^{T} \Gamma \Phi(\mathbf{x})\right] \\
& \leq\left(\lambda_{3}+2 L \sqrt{\frac{\lambda_{2}}{\lambda_{1}}}\right) V(\mathbf{x}), \quad t \neq \tau_{k}
\end{aligned}
$$

Hence, condition 1 of Theorem 1 is satisfied with $g(t, w)=$ $\left(\lambda_{3}+2 L \sqrt{\lambda_{2} / \lambda_{1}}\right) w$.

Since $B$ is symmetric, we know that $I+B$ is also symmetric. By using Euclidean norm we have

$$
\rho(I+B)=\|I+B\| .
$$

Given any $\rho_{0}>0$ and $\mathrm{x} \in S_{\rho_{0}}$, we have

$$
\begin{aligned}
\|\mathbf{x}+U(k, \mathbf{x})\| & =\|\mathbf{x}+B \mathbf{x}\| \leq\|I+B\|\|\mathbf{x}\| \\
& =\rho(I+B)\|\mathbf{x}\| \leq\|\mathbf{x}\| .
\end{aligned}
$$

The last inequality is in view of $\rho(I+B) \leq 1$. From which we know that $\mathbf{x}+U(k, \mathbf{x}) \in S_{\rho_{0}}$. 


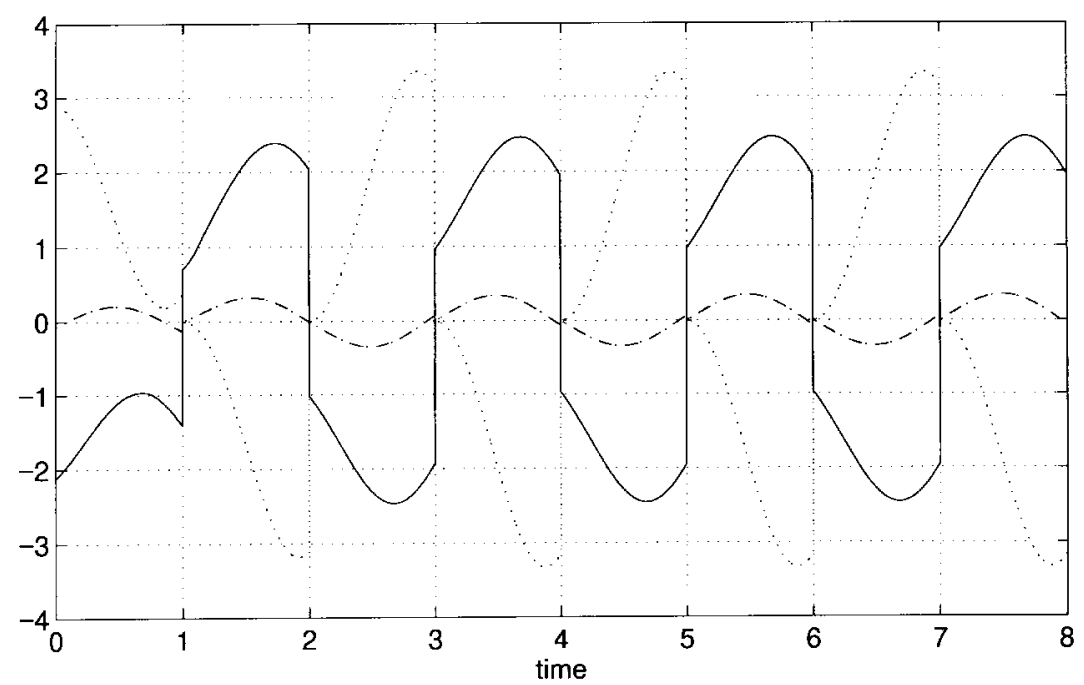

(a)

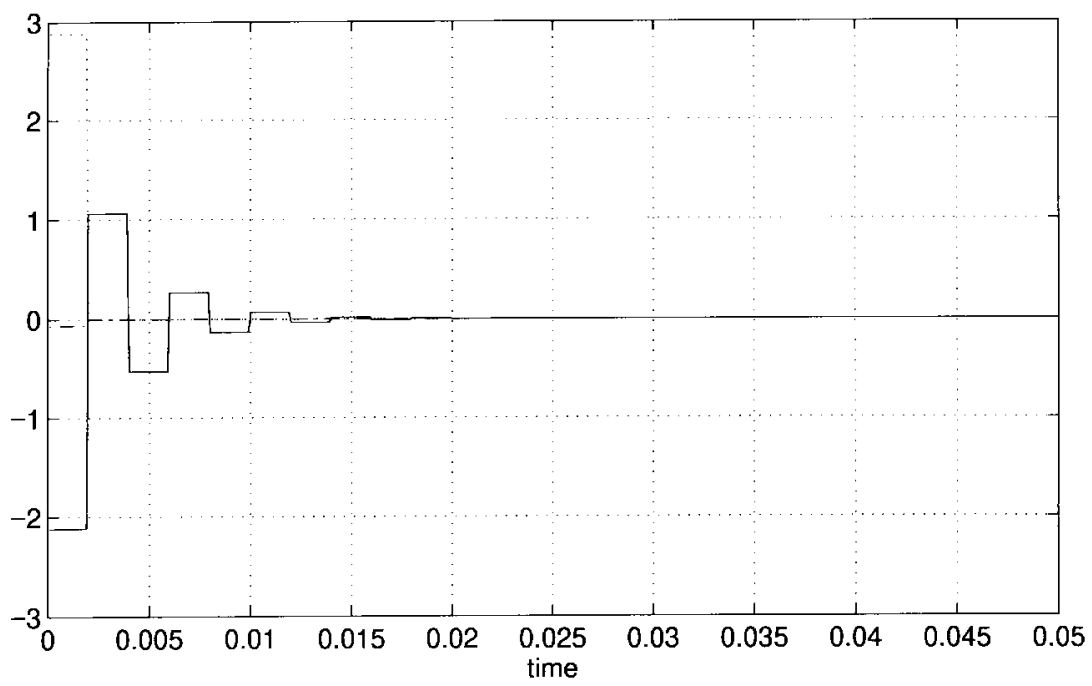

(b)

Fig. 3. Simulation results. (a) Unstable results outside the stable region. (b) Stable results inside the predicted stable region.

When $t=\tau_{k}$, we have

$$
\begin{aligned}
\left.V(\mathbf{x}+B \mathbf{x})\right|_{t=\tau_{k}} & =(\mathbf{x}+B \mathbf{x})^{T} \Gamma(\mathbf{x}+B \mathbf{x}) \\
& =\mathbf{x}^{T}\left[I+(B)^{T}\right] \Gamma(I+B) \mathbf{x} \\
& \leq \lambda_{4} V(\mathbf{x}) .
\end{aligned}
$$

Hence, condition 2 of Theorem 1 is satisfied with $\psi_{k}(w)=$ $\lambda_{4} w$. And

$$
\lambda_{1}\|\mathbf{x}\|^{2} \leq V(\mathbf{x}) \leq \lambda_{2}\|\mathbf{x}\|^{2} .
$$

From which one can see that condition 3 of Theorem 1 is also satisfied with $\beta(x)=\lambda_{1} x$ and $\alpha(x)=\lambda_{2} x$. It follows from Theorem 1 that the asymptotic stability of the impulsive control system in (18) is implied by that of the following comparison system:

$$
\left\{\begin{array}{l}
\dot{w}(t)=\left(\lambda_{3}+2 L \sqrt{\frac{\lambda_{2}}{\lambda_{1}}}\right) w(t), \quad t \neq \tau_{k} \\
w\left(\tau_{k}^{+}\right)=\lambda_{4} w\left(\tau_{k}\right) \\
w\left(t_{0}^{+}\right)=w_{0} \geq 0
\end{array} .\right.
$$

It follows from Theorem 2 that if

$$
\int_{\tau_{k}}^{\tau_{k+1}}\left(\lambda_{3}+2 L \sqrt{\frac{\lambda_{2}}{\lambda_{1}}}\right) d t+\ln \left(\gamma \lambda_{4}\right) \leq 0, \quad \gamma>1
$$

i.e.,

$$
\left(\lambda_{3}+2 L \sqrt{\frac{\lambda_{2}}{\lambda_{1}}}\right)\left(\tau_{k+1}-\tau_{k}\right) \leq-\ln \left(\gamma \lambda_{4}\right), \quad \gamma>1
$$
and

$$
\lambda_{3}+2 L \sqrt{\frac{\lambda_{2}}{\lambda_{1}}} \geq 0
$$

are satisfied, then the origin of (18) is asymptotically stable.

Since this lemma is too general, in practical application, we want to deal with a simple case which is given in the following theorem.

We use the following theorem in order to guarantee the asymptotic stability of the origin of the controlled Chua's oscillator. 


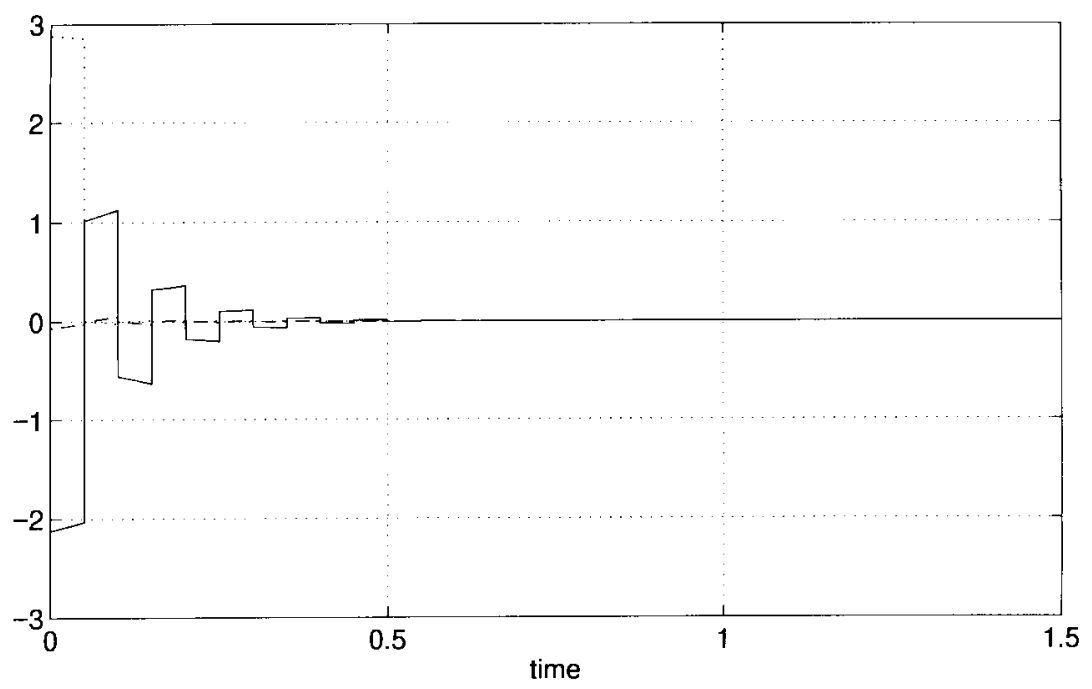

(c)

Fig. 3. (Continued.) Simulation results. (c) Stable results outside the predicted stable region.

Theorem 3: Let $d_{1}$ be the largest eigenvalue of $(I+$ $\left.B^{T}\right)(I+B)$, where $B$ is a symmetric matrix, $\rho(I+B) \leq 1$, where $\rho(\cdot)$ denotes the spectral radius of $I+B$. Let $q$ be the largest eigenvalue of $\left(A+A^{T}\right)$ and let the impulses be equidistant from each other and separated by interval $\Delta$. If

$$
0 \leq q+2|\alpha a| \leq-\frac{1}{\Delta} \ln \left(\xi d_{1}\right), \quad \text { where } \xi>1
$$

then the origin of the impulsively controlled Chua's oscillator is asymptotically stable.

Proof: Let us construct the Lyapunov function $V(t, \mathbf{x})=$ $\mathbf{x}^{T} \mathbf{x}$. For $t \neq \tau_{i}$, we have

$$
\begin{aligned}
D^{+} V(t, \mathrm{x}) & =\mathrm{x}^{T} A \mathbf{x}+\mathbf{x}^{T} A^{T} \mathbf{x}+\mathbf{x}^{T} \Phi(\mathbf{x})+\Phi^{T}(\mathbf{x}) \mathbf{x} \\
& \leq q \mathbf{x}^{T} \mathbf{x}+2|\alpha a| \mathbf{x}^{T} \mathbf{x} \\
& =(q+2|\alpha a|) V(t, \mathbf{x})
\end{aligned}
$$

Hence, condition 1 of Theorem 1 is satisfied with $g(t, w)=$ $(q+2|\alpha a|) w$.

Since $B$ is symmetric, we know $(I+B)$ is also symmetric. By using Euclidean norm we have

$$
\rho(I+B)=\|I+B\| .
$$

Given any $\rho_{0}>0$ and $\mathrm{x} \in S_{\rho_{0}}$, we have

$$
\|\mathbf{x}+B \mathbf{x}\| \leq\|I+B\|\|\mathbf{x}\|=\rho(I+B)\|\mathbf{x}\| \leq\|\mathbf{x}\| .
$$

The last inequality follows from $\rho(I+B) \leq 1$. Consequently, $\mathbf{x}+B \mathbf{x} \in S_{p_{0}}$.

For $t=\tau_{i}$, we have

$$
\begin{aligned}
V\left(\tau_{i}, \mathbf{x}+B \mathbf{x}\right) & =(\mathbf{x}+B \mathbf{x})^{T}(\mathbf{x}+B \mathbf{x}) \\
& =\mathbf{x}^{T}\left(I+B^{T}\right)(I+B) \mathbf{x} \\
& \leq d_{1} V\left(\tau_{i}, \mathbf{x}\right) .
\end{aligned}
$$

Hence, condition 2 of Theorem 1 is satisfied with $\psi_{i}(w)=$ $d_{1} w$. We can see that condition 3 of Theorem 1 is also satisfied. It follows from Theorem 1 that the asymptotic stability of the impulsively controlled Chua's oscillator in (18) is implied by that of the following comparison system

$$
\left\{\begin{array}{l}
\dot{\omega}=(q+2|\alpha a|) \omega, \quad t \neq \tau_{i} \\
\omega\left(\tau_{i}\right)=d_{1} \omega\left(\tau_{i}\right) \\
\omega\left(t_{0}\right)=\omega_{0} \geq 0
\end{array} .\right.
$$

From (32), we have

$$
\int_{\tau_{i}}^{\tau_{i+1}}(q+2|\alpha a|) d t+\ln \left(\xi d_{1}\right) \leq 0, \quad \xi>1
$$

and $\dot{\lambda}(t)=q+2|\alpha a| \geq 0$. It follows from Theorem 2 that the trivial solution of (18) is asymptotically stable.

Theorem 3 also gives an estimate for the upper bound $\Delta_{\max }$ of $\Delta$; namely,

$$
\Delta_{\max }=\left|\frac{\ln \left(\xi d_{1}\right)}{q+2|\alpha a|}\right|, \quad \xi \longrightarrow 1^{+} .
$$

Observe that the upper bound given by (39) is sufficient but not necessary. Consequently, we can only say that we have a predicted stable region, which is usually smaller than the actual stable region because we can not assert that all other regions are unstable.

\section{Simulation Results of Impulsive Control}

In the following simulations, we choose the parameters of Chua's oscillator as $\alpha=15, \beta=20, \gamma=0.5, a=\frac{120}{7}, b=$ $-\frac{75}{7}$. A fourth-order Runge-Kutta method with step size $10^{-5}$ is used. The initial condition is given by $[x(0), y(0), z(0)]$ $=(-2.121304,-0.066170,2.881090)$. The uncontrolled trajectories are shown in Fig. 1, which is the Chua's double scroll attractor.

\section{A. Simulation 1: Strong Control}

In this simulation, we choose the matrix $B$ as

$$
B=\left(\begin{array}{rrr}
k & 0 & 0 \\
0 & -1 & 0 \\
0 & 0 & -1
\end{array}\right)
$$


where the impulsive control is "strong." It follows from Theorem 3 that $\rho(I+B) \leq 1$ should be satisfied, which implies that $-2 \leq k \leq 0$. By using this $B$ matrix, it is easy to see that

$$
d_{1}=(k+1)^{2} .
$$

We have

$$
\begin{aligned}
A & =\left(\begin{array}{ccc}
-15 & 15 & 0 \\
1 & -1 & 1 \\
0 & -20 & -0.5
\end{array}\right), \\
A+A^{T} & =\left(\begin{array}{ccc}
-30 & 16 & 0 \\
16 & -2 & -19 \\
0 & -19 & -1
\end{array}\right)
\end{aligned}
$$

from which we find $q=20.162180$. Then an estimate of the boundaries of the stable region is given by

$$
0 \leq \Delta \leq-\frac{\left[\ln \xi+\ln (k+1)^{2}\right]}{q+2|\alpha a|}, \quad-2 \leq k \leq 0 .
$$

Fig. 2 shows the stable region for different $\xi$ 's. The entire region below the curve corresponding to $\xi=1$ is the predicted stable region. When $\xi \longrightarrow \infty$, the stable region shrinks to a line $k=-1$.

The simulation results are shown in Fig. 3. Fig. 3(a) shows instability for $k=-1.5$ and $\Delta=1$. The solid waveform, the dash-dotted waveform, and the dotted waveform correspond to $x(t), y(t)$, and $z(t)$, respectively. Fig. 3(b) shows stable results within the stable region for $k=-1.5$ and $\Delta=0.002$. One can see that the system asymptotically approaches the origin with a settling time of about 0.05 . However, the true stable region is larger than that predicted in Fig. 2. In order to demonstrate this fact, we show in Fig. 3(c) the stable results for $k=-1.5$ and $\Delta=0.05$. We can also see that the system asymptotically approaches the origin with a settling time of about 1.4 which is much larger than that shown in Fig. 3(b).

\section{B. Simulation 2: Weak Control}

In this simulation, we choose the matrix $B$ as

$$
B=\left(\begin{array}{ccc}
k & 0 & 0 \\
0 & -0.1 & 0 \\
0 & 0 & -0.1
\end{array}\right)
$$

where the impulsive control is much weaker than that chosen in simulation 1 .

It is easy to see that

$$
d_{1}= \begin{cases}(k+1)^{2}, & (k+1)^{2} \geq 0.81 \\ 0.81, & \text { elsewhere }\end{cases}
$$

An estimate of the boundaries of the stable region is given by

$$
0 \leq \Delta \leq \begin{cases}-\frac{\ln \xi+\ln (k+1)^{2}}{q+2|\alpha a|} & (k+1)^{2} \geq 0.81 \\ -\frac{\ln \xi+\ln (0.81)}{q+2|\alpha a|} & \text { elsewhere } \\ -2 \leq k \leq 0 & \end{cases}
$$

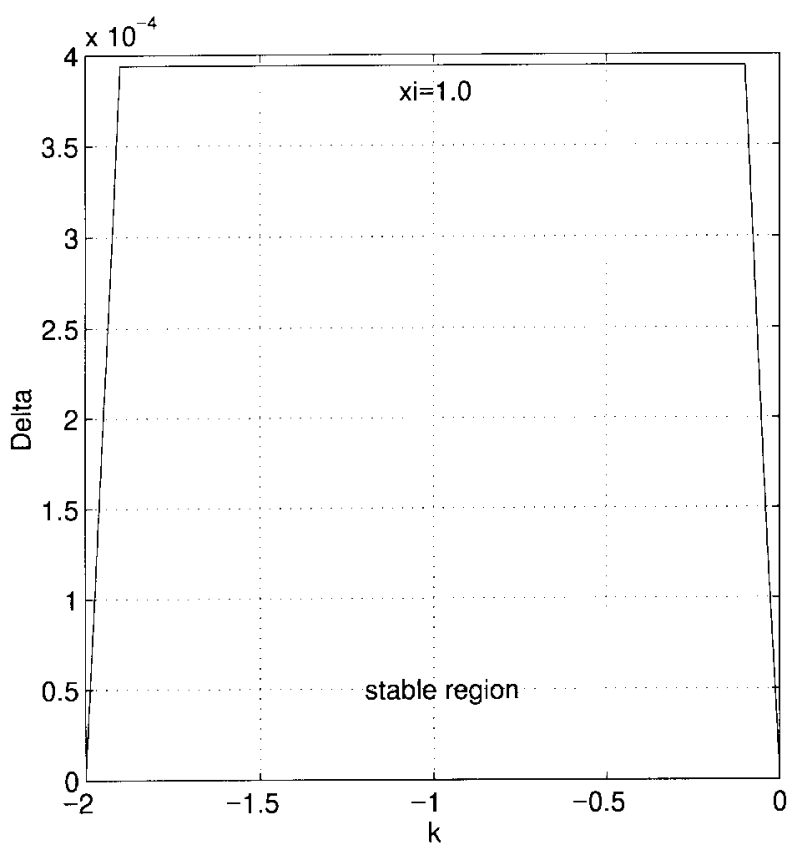

Fig. 4. Estimate of the boundaries of stable region used in simulation 2.

Fig. 4 shows the stable region. The entire region below the curve corresponding to $\xi=1$ is the predicted stable region. In this case, $\Delta$ is always bounded. It seems that we can't control the system to the origin with an arbitrarily prescribed speed because $\xi$ has to satisfy $1<\xi<\frac{100}{81}$. This is different from the case shown in Fig. 2, where any value of $\xi>1$ is possible.

The simulation results are shown in Fig. 5. Again, the solid waveform, the dash-dotted waveform, and the dotted waveform correspond to $x(t), y(t)$, and $z(t)$, respectively. Fig. 5(a) shows the instability results for $k=-1$ and $\Delta=$ 0.4. Fig. 5(b) shows the stable results in the stable region for $k=-1$ and $\Delta=3 \times 10^{-4}$. The control system asymptotically approaches the origin with a settling time of about 0.05 . Also, the true stable region is larger than that predicted in Fig. 4. To demonstrate this fact, we show in Fig. 5(c) the stable results for $k=-1$ and $\Delta=0.01$. We can also see that the system asymptotically approaches the origin with a settling time equal approximately to 1 , which is much larger than that shown in Fig. 5(b).

\section{SYNCHRONIZATION OF CHUA'S OSCILLATORS USING IMPULSIVE CONTROL}

In this section, we study the impulsive synchronization of two Chua's oscillators. One of the Chua's oscillators is called the driving system and the other is called the driven system. In an impulsive synchronization configuration, the driving system is given by (14). The driven system is given by

$$
\dot{\tilde{\mathbf{x}}}=A \tilde{\mathbf{x}}+\Phi(\tilde{\mathbf{x}})
$$

where $\tilde{\mathbf{x}}^{T}=(\tilde{x}, \tilde{y}, \tilde{z})$ is the state variables of the driven system.

At discrete instants, $\tau_{i}, i=1,2, \cdots$, the state variables of the driving system are transmitted to the driven system and then the state variables of driven system are subject to jumps 


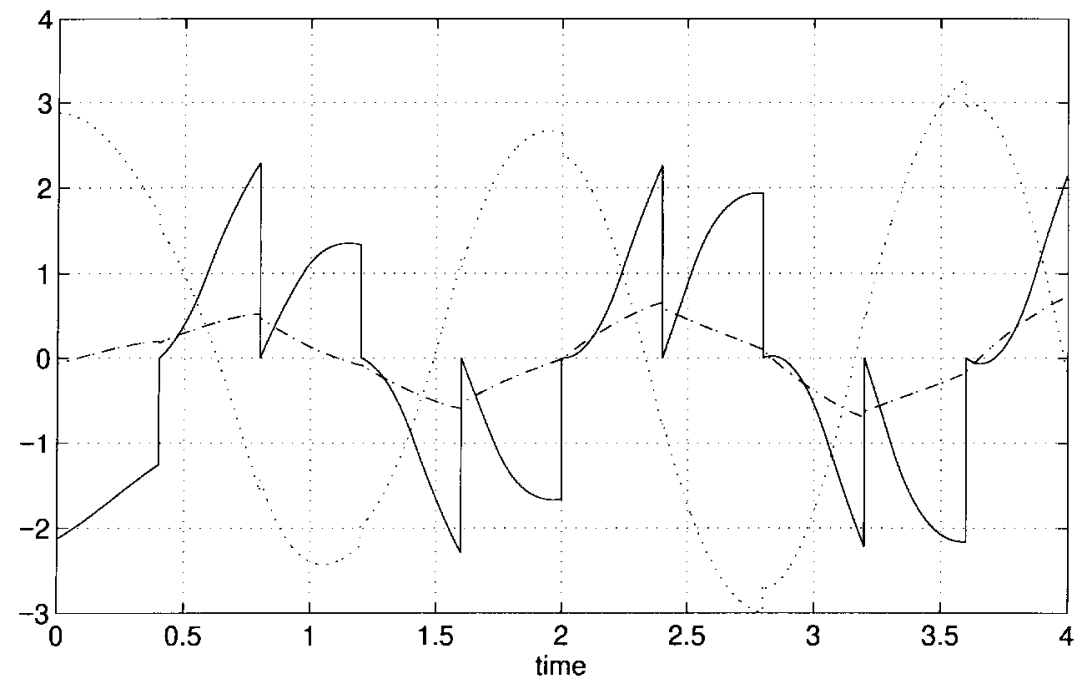

(a)

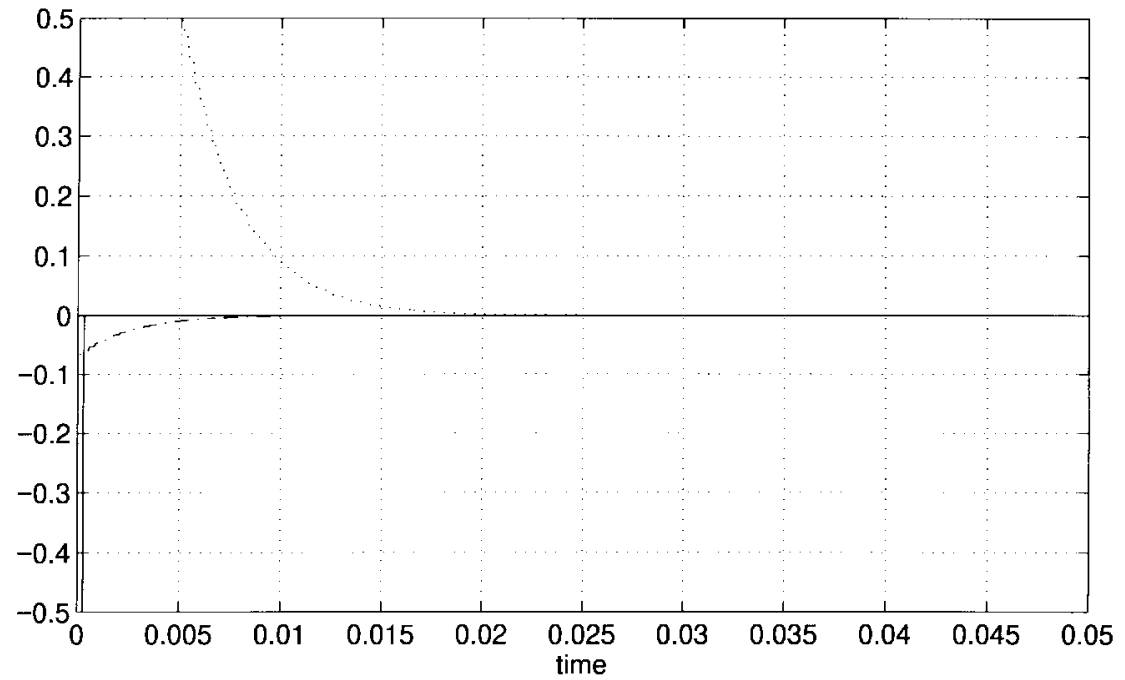

(b)

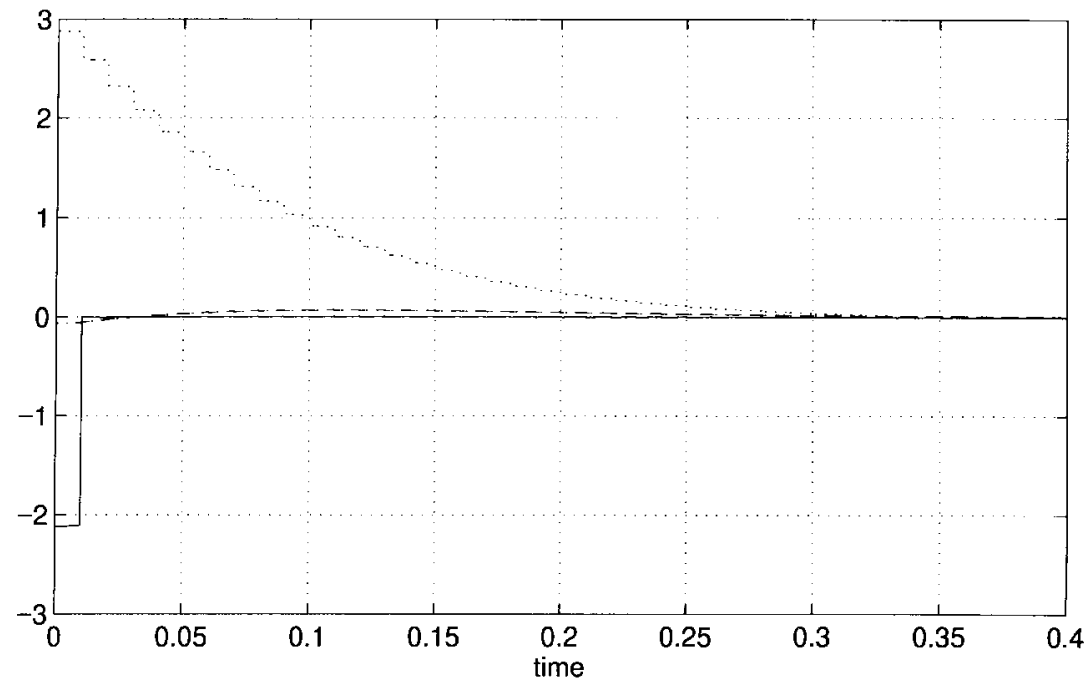

(c)

Fig. 5. Simulation results. (a) Unstable results outside the stable region. (b) Stable results in the stable region. (c) Stable results outside the stable region. 


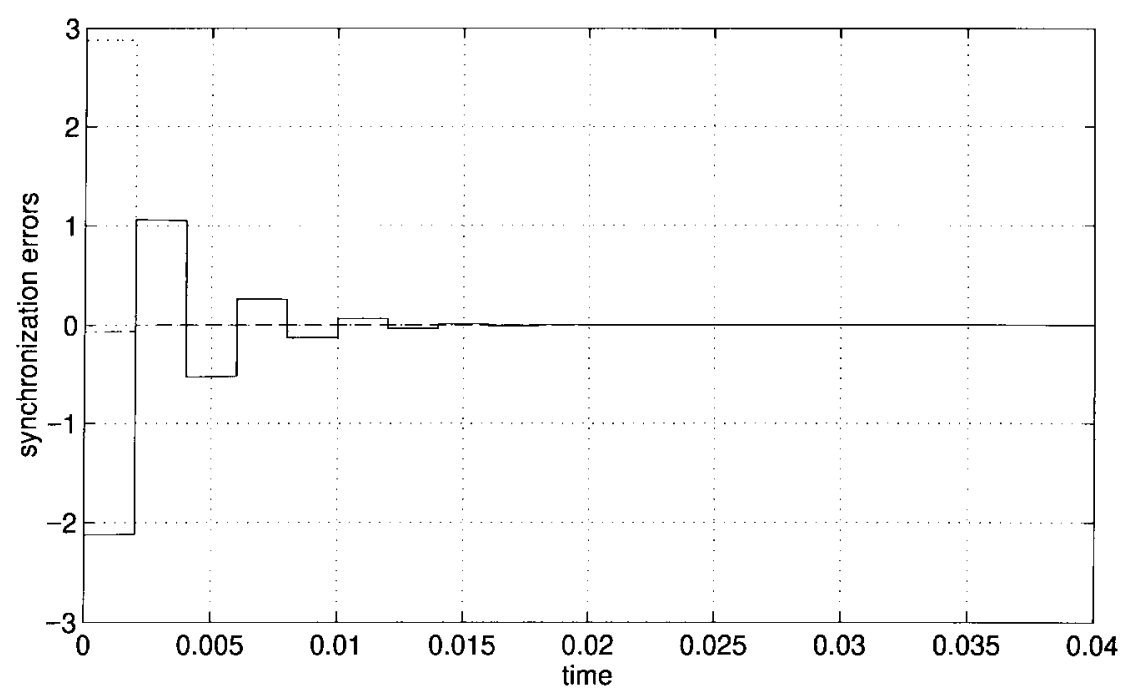

(a)

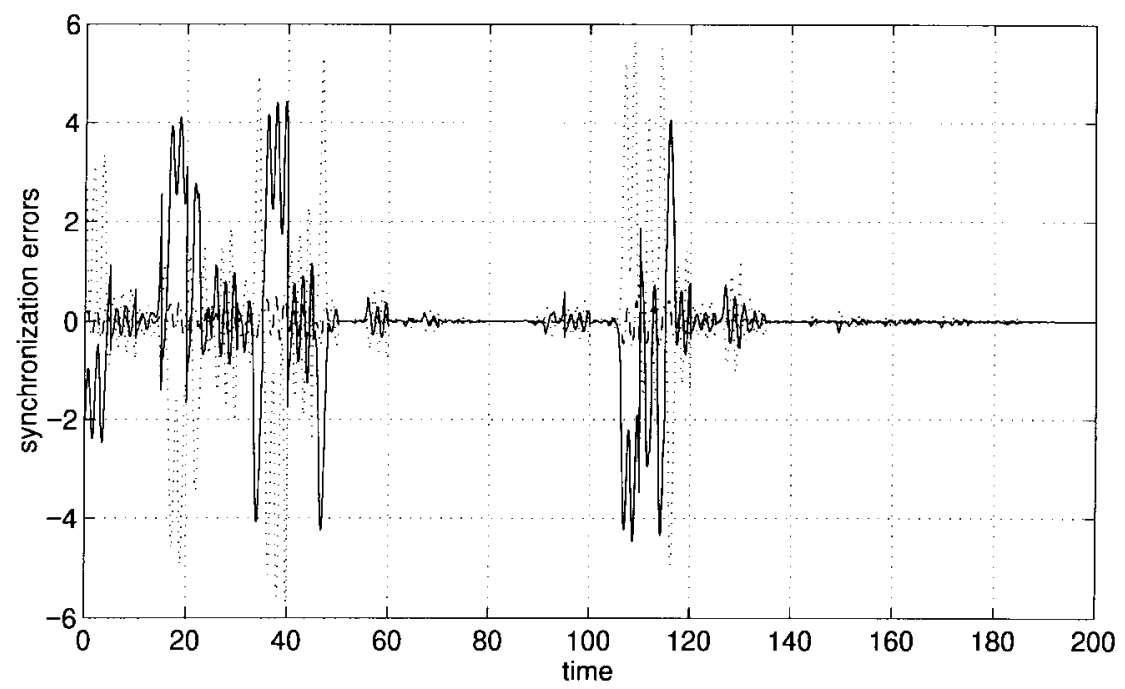

(b)

Fig. 6. Simulation results of impulsive synchronization: (a) stable synchronization results inside our predicted stable region and (b) synchronization can not be achieved when $\Delta$ is too large.

at these instants. In this sense, the driven system is described by the impulsive differential equation

$$
\left\{\begin{array}{l}
\dot{\tilde{\mathbf{x}}}=A \tilde{\mathbf{x}}+\Phi(\tilde{\mathbf{x}}), \quad t \neq \tau_{i} \\
\left.\Delta \tilde{\mathbf{x}}\right|_{t=\tau_{i}}=-B \mathbf{e}, \quad i=1,2, \cdots
\end{array}\right.
$$

where $B$ is a $3 \times 3$ matrix, and $\mathbf{e}^{T}=\left(e_{x}, e_{y}, e_{z}\right)=(x-\tilde{x}$, $y-\tilde{y}, z-\tilde{z})$ is the synchronization error. If we define

$$
\Psi(\mathbf{x}, \tilde{\mathbf{x}})=\Phi(\mathbf{x})-\Phi(\tilde{\mathbf{x}})=\left[\begin{array}{c}
-\alpha f(x)+\alpha f(\tilde{x}) \\
0 \\
0
\end{array}\right]
$$

then the error system of the impulsive synchronization is given by

$$
\begin{cases}\dot{\mathbf{e}}=A \mathbf{e}+\Psi(\mathbf{x}, \tilde{\mathbf{x}}), & t \neq \tau_{i} \\ \left.\Delta \mathbf{e}\right|_{t=\tau_{i}}=B \mathbf{e}, & i=1,2, \ldots\end{cases}
$$

We use the following theorem to guarantee that our impulsive synchronization is asymptotically stable.
Theorem 4: Let $d_{1}$ be the largest eigenvalue of $(I+$ $\left.B^{T}\right)(I+B)$, where $B$ is a symmetric matrix. Assume the spectral radius $\rho$ of $I+B$ satisfies $\rho(I+B) \leq 1$. Let $q$ be the largest eigenvalue of $\left(A+A^{T}\right)$ and assume the impulses are equidistant from each other and separated by an interval $\Delta$. If

$$
0 \leq q+2|\alpha a| a \leq-\frac{1}{\Delta} \ln \left(\xi d_{1}\right), \quad \xi>1
$$

then the impulsive synchronization of two Chua's oscillators is asymptotically stable.

Proof: Observe that the error system in (50) is almost the same as the system in (18) except for $\Psi(\mathbf{x}, \tilde{\mathbf{x}})$. Similarly, let us construct the Lyapunov function $V(t, \mathbf{e})=\mathbf{e}^{T} \mathbf{e}$. For $t \neq \tau_{i}$, we have

$$
\begin{aligned}
D^{+} V(t, \mathbf{e}) & =\mathbf{e}^{T} A \mathbf{e}+\mathbf{e}^{T} A^{T} \mathbf{e}+\mathbf{e}^{T} \Psi(\mathbf{e})+\Psi^{T}(\mathbf{e}) \mathbf{e} \\
& \leq q \mathbf{e}^{T} \mathbf{e}+2|\alpha||f(x)-f(\tilde{x})| e_{x} \\
& \leq q \mathbf{e}^{T} \mathbf{e}+2|\alpha a| e_{x}^{2}
\end{aligned}
$$




$$
\begin{aligned}
& \leq(q+2|\alpha a|) \mathbf{e}^{T} \mathbf{e} \\
& =(q+2|\alpha a|) V(t, \mathbf{e}) .
\end{aligned}
$$

Hence, condition 1 of Theorem 1 is satisfied with $g(t, w)=$ $(q+2|\alpha a|) w$. The rest of this proof is the same as that of Theorem 3.

For the rest of this section, we present our simulation results. We choose the matrix $B$ as

$$
B=\left(\begin{array}{rrr}
-1.5 & 0 & 0 \\
0 & -1 & 0 \\
0 & 0 & -1
\end{array}\right) \text {. }
$$

The initial conditions are given by $[x(0), y(0), z(0)]=$ $(-2.121304,-0.066170,2.881090)$ and $[\tilde{x}(0), \tilde{y}(0), \tilde{z}(0)]$ $=(0,0,0)$. The other parameters are the same as those used in Section IV. Since the stability boundary estimates are the same as those in Section IV, we do not repeat them here. Fig. 6 shows our simulation results. Fig. 6(a) shows the stable results within our predicted stable region with $k=-1.5$ and $\Delta=0.002$. The solid line, the dash-dotted line, and the dotted line show $e_{x}(t), e_{y}(t)$, and $e_{z}(t)$, respectively. We can see that impulsive synchronization was achieved rapidly. Fig. 6(b) shows that if $\Delta=5$ then our impulsive synchronization is unstable.

\section{APPLICATION OF IMPULSIVE Synchronization to SECURE COMMUNICATION}

Since the publication of several chaotic cryptanalysis results of low-dimensional chaos-based secure communication systems [12], [13], there existed an illusion that such communication schemes were not secure enough. It may be reasonable to exploit hyperchaos based secure communication systems, but such systems may introduce more difficulties to synchronization.

On the other hand, we can enhance the security of lowdimensional chaos-based secure communication schemes by combining conventional cryptographic schemes with a chaotic system [14]. To overcome the low security objections against low-dimensional continuous chaos-based schemes, we should overcome the following problems: 1) make the transmitted signal more complex and 2) reduce the redundancy in the transmitted signal. To achieve the first goal, it is not necessary to use hyper-chaos. In [14], we have presented a method to combine a conventional cryptographic scheme with lowdimensional chaos to obtain a very complex transmitted signal. To achieve the second goal, impulsive synchronization offers a very promising approach.

In this section, we combine the results in [14] and impulsive synchronization to give a new chaotic secure communication scheme. The block diagram of this scheme is shown in Fig. 7.

From Fig. 7, we can see that this chaotic secure communication system consists of a transmitter and a receiver. In both the transmitter and the receiver, there exist two identical chaotic systems. Also, two identical conventional cryptographic schemes are embedded in both the transmitter and the receiver. Let us now consider details of each block
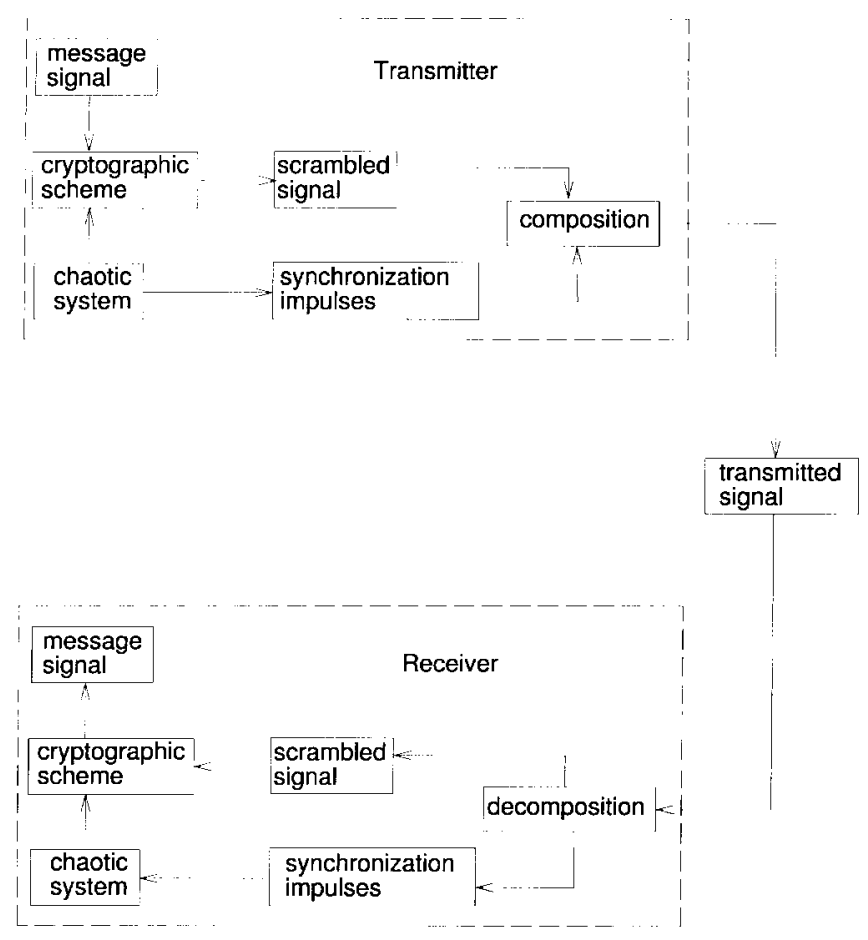

Fig. 7. Block diagram of the impulsive-synchronization based chaotic secure communication system.

in Fig. 7. The transmitted signal consists of a sequence of time frames. Every frame has a length of $T$ seconds and consists of two regions. In Fig. 8, we show the concept of a time frame and its components. The first region of the time frame is a synchronization region consisting of synchronization impulses. The synchronization impulses are used to impulsively synchronize the chaotic systems in both transmitter and receiver. The second region is the scrambled signal region where the scrambled signal is contained. To ensure synchronization, we have $T<\Delta_{\max }$. Within every time frame, the synchronization region has a length of $Q$ and the remaining time interval $T-Q$ is the scrambled signal region.

The composition block in Fig. 7 is used to combine the synchronization impulses and the scrambled signal into the time frame structure shown in Fig. 8. The simplest combination method is to substitute the beginning $Q$ seconds of every time frame with synchronization impulses. Since $Q$ is usually very small compared with $T$, the loss of time for packing a message signal is negligible. The decomposition block is used to separate the synchronization region and the scrambled signal region within each frame at the receiver end. Then the separated synchronization impulses are used to make the chaotic system in the receiver to synchronize with that in the transmitter. The stability of this impulsive synchronization is guaranteed by our results in Section V.

In the transmitter and the receiver, we use the same cryptographic scheme block for purposes of bidirectional communication. In a bidirectional communication scheme, every cellular phone should function both as a receiver and a 


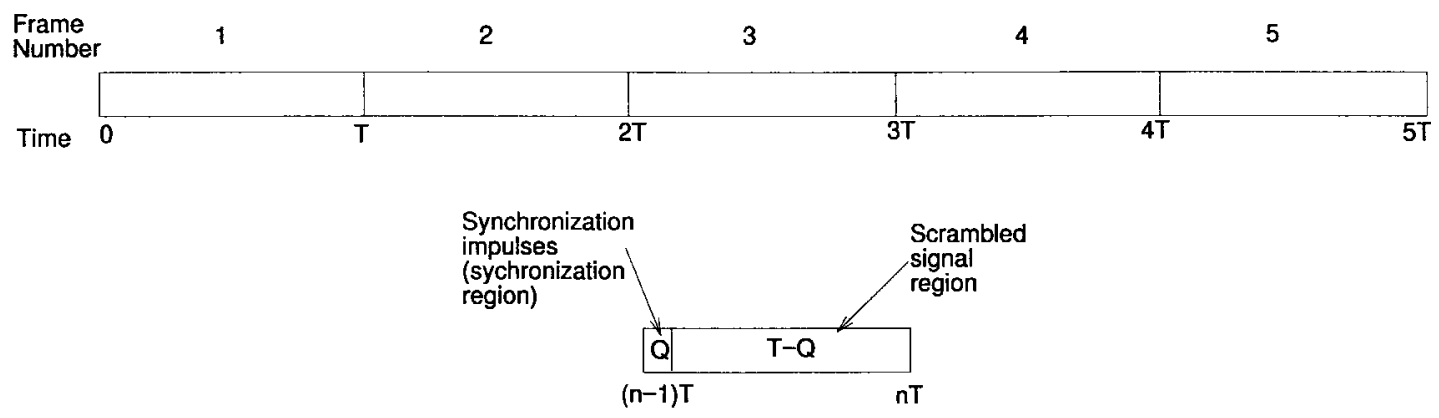

Fig. 8. Illustration of the concept of a time-frame and its components.

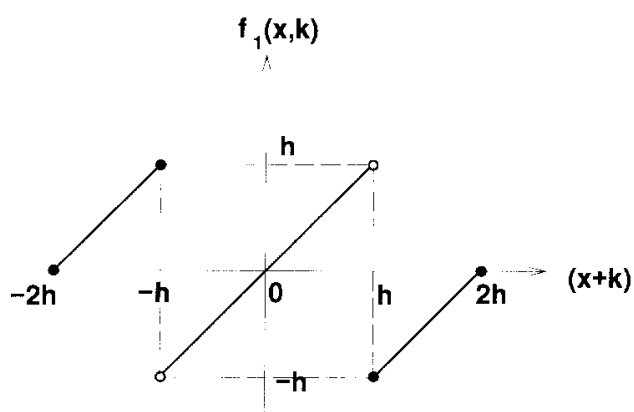

Fig. 9. Nonlinear function used in the continuous shift cipher.

transmitter. Here, the key signal is generated by the chaotic system. The cryptographic scheme is as follows [14]:

We use a continuous $n$-shift cipher to encrypt the plain signal(message signal). The $n$-shift cipher is given by

$$
\begin{aligned}
e[p(t)] & =\underbrace{f_{1}\left(\cdots f _ { 1 } \left\{f_{1}\right.\right.}_{n}[p(t), \underbrace{k(t)], k(t)\}, \cdots, k(t))}_{n} \\
& =y(t)
\end{aligned}
$$

where $h$ is chosen such that $p(t)$ and $k(t)$ lie within $(-h, h)$. Here, $p(t)$ and $k(t)$ denote the plain signal and the key signal, respectively, and $y(t)$ denotes the encrypted signal. The key signal $k(t)$ is chosen as a state variable of the chaotic system. The notation $f_{1}(\cdot, \cdot)$ denotes a scalar nonlinear function of two variables defined as follows:

$$
f_{1}(x, k)=\left\{\begin{array}{lc}
(x+k)+2 h, & -2 h \leq(x+k) \leq-h \\
(x+k), & -h<(x+k)<h \\
(x+k)-2 h, & h \leq(x+k) \leq 2 h
\end{array} .\right.
$$

This function is shown in Fig. 9.

The corresponding decryption rule is the same as the encryption rule

$$
\begin{aligned}
p(t) & =d[y(t)] \\
& =\underbrace{f_{1}\left(\cdots f _ { 1 } \left\{f_{1}\right.\right.}_{n}[y(t), \underbrace{-k(t)],-k(t)\}, \cdots,-k(t))}_{n} .
\end{aligned}
$$

To decode the encrypted signal, the same key signal should be used.
The simulation results are as follows. We use an FM scheme to modulate the synchronization impulses such that the synchronization region is located in the initial $1 \%$ of every time frame. We choose the frame length as $T=1 \mathrm{~s}$. In the synchronization region of every time frame, we transmit the impulses of the three state variables of the Chua's oscillators. The parameter of the encrypted signal is chosen as $h=0.4$. A continuous 10-shift cipher was used. We choose $x$ and $\tilde{x}$ as the key signals and normalized them to fall within the amplitude range $[-0.4,0.4]$.

Fig. 10 shows the simulation results of the above proposed secure communication system for transmitting a speech signal. Fig. 10(a) shows the waveforms of the sampled speech of four Chinese digits "NING"(zero)_-YY"(one)"ER"(two)-“SANG"(three). The sampling rate is 8k. Fig. 10(b) shows the spectrograms of the original speech signal in Fig. 10(a), from which we can see the structure of the speech signal. Fig. 10(c) shows the waveforms of the received scrambled speech signal and the additive channel noise with $\mathrm{SNR}=16 \mathrm{~dB}$. This additive noise cannot change the value of the synchronization impulses which are modulated by FM. Fig. 10(d) shows the spectrograms for the scrambled speech signal and the additive channel noise. We can see that the structure of the signal in Fig. 10(b) was totally covered by an almost uniformly distributed noise-like spectrum. Fig. 10(e) shows the waveforms of the descrambled speech signal. Fig. 10(f) shows the spectrograms of the descrambled speech signal. We can see that some noises were introduced into the recovered results due to the channel noise, and that the spectrograms became a little blurry. But the structure of the speech signal was perfectly recovered.

\section{CONCLUDING REMARKS}

In this paper, we have presented a theory of impulsive control of chaotic dynamical systems. An estimate of the upper bound of the impulse interval $\Delta$ is also presented. Since all of our results are based on rigorous theoretical analysis and proofs, the results in this paper provide a framework and foundation for future works. We then use this theory to impulsively control and synchronize Chua's oscillators. An application of impulsive chaotic synchronization to secure communication is presented. The chaotic secure communication scheme presented here is a combination of a conventional cryptographic method and impulsive synchronization. 


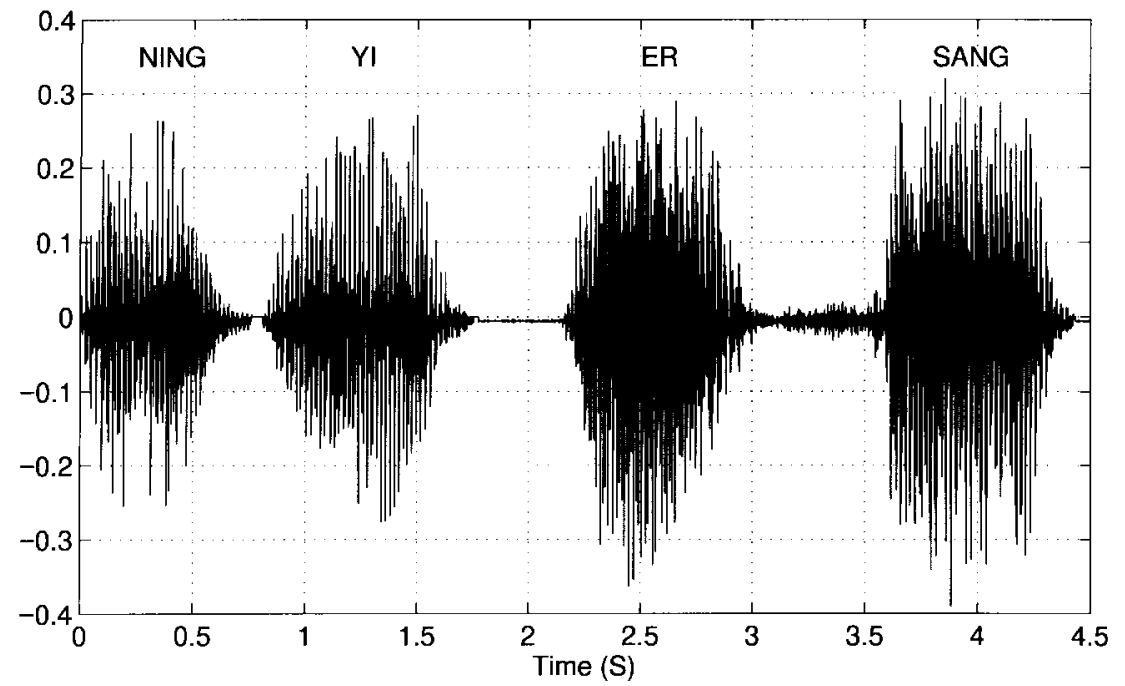

(a)

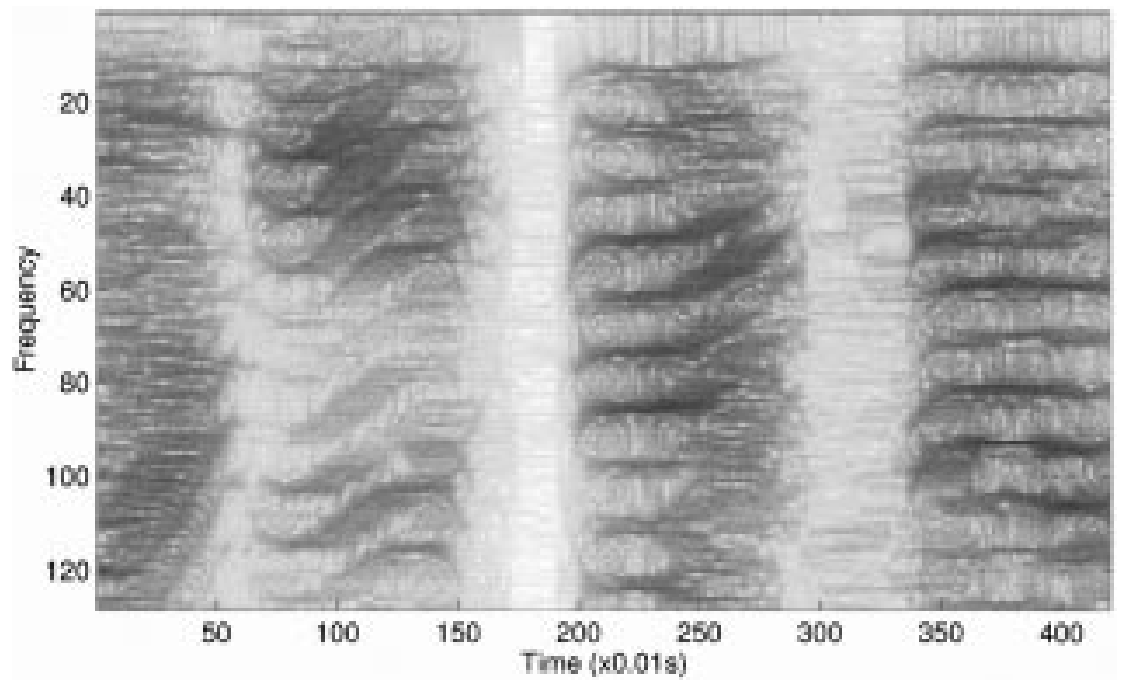

(b)

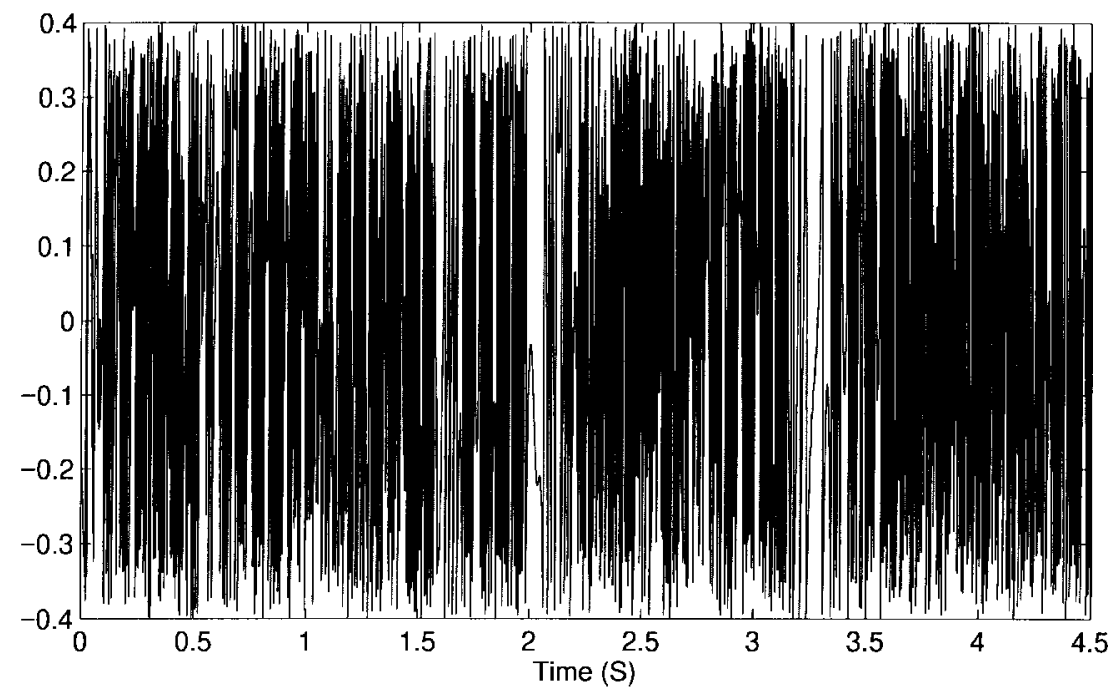

(c)

Fig. 10. The simulation results. (a) The time-domain waveform of the speech signal. (b) The spectrogram for the original speech signal. (c) The time-domain waveform of the scrambled speech signal. 


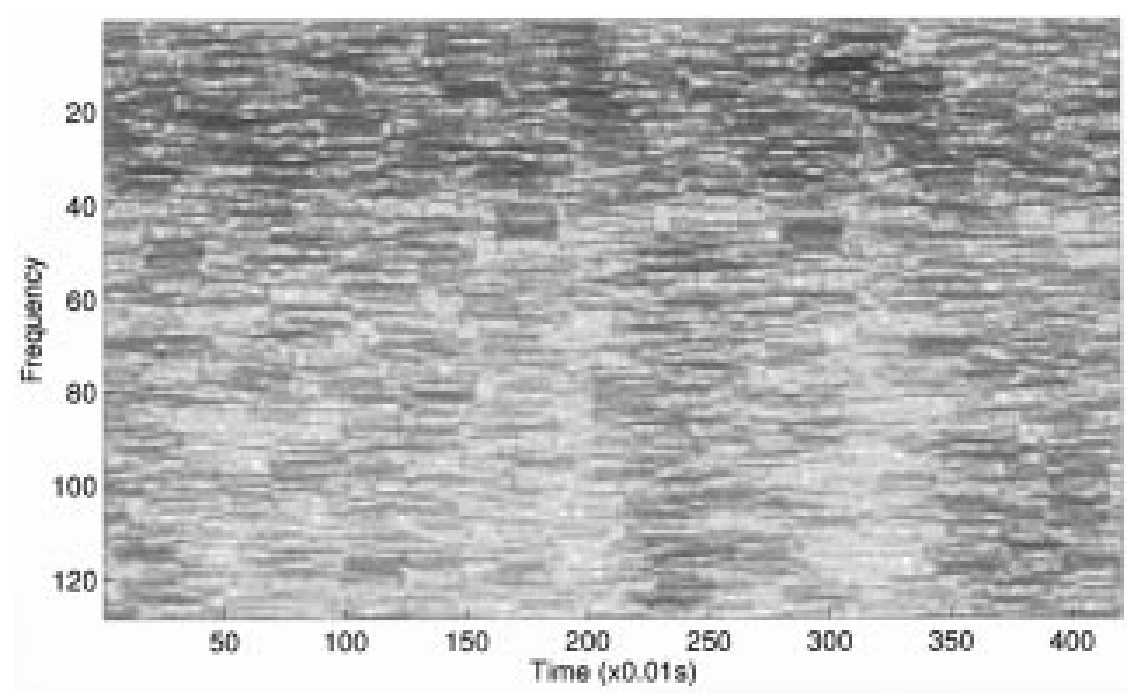

(d)

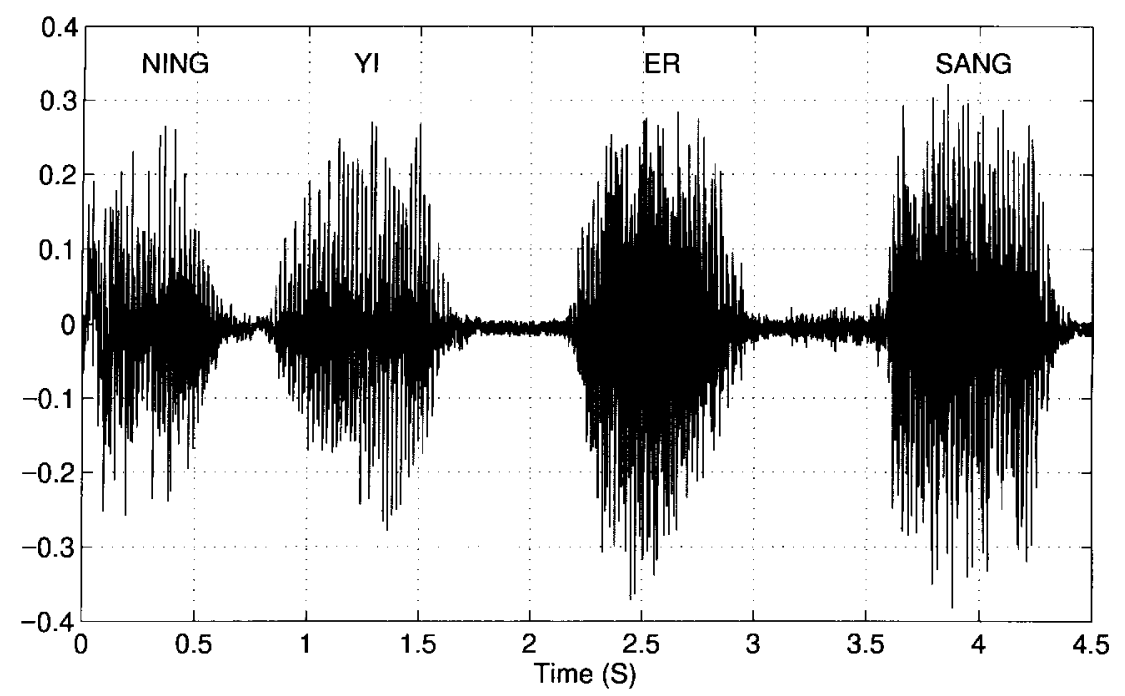

(e)

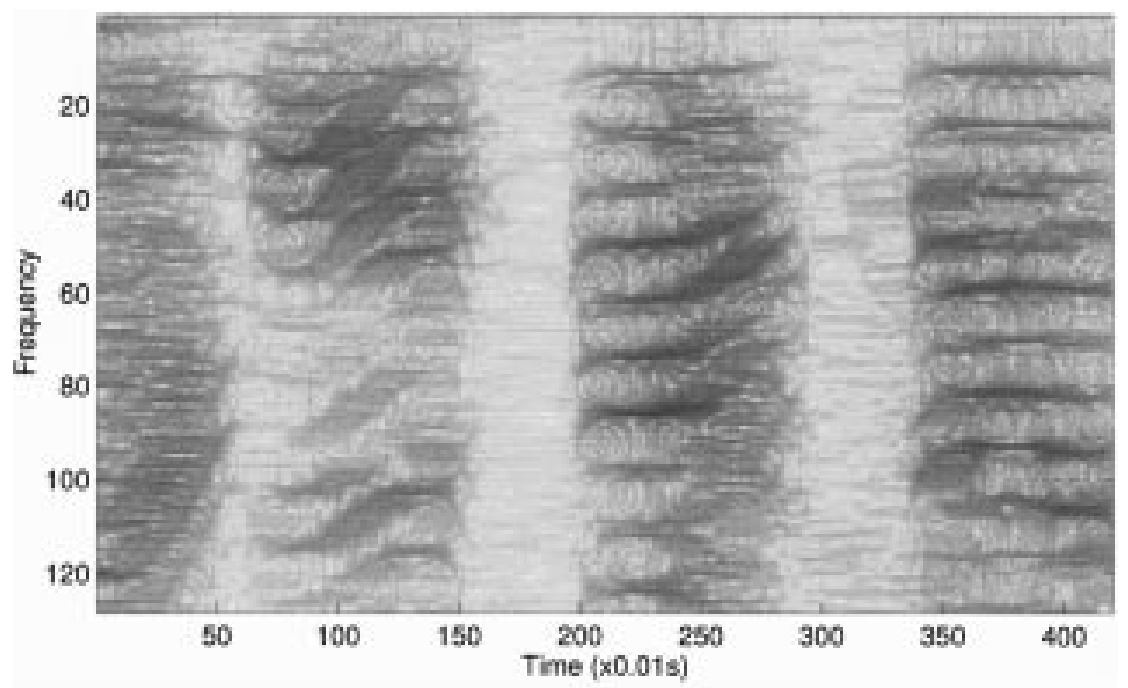

(f)

Fig. 10. (Continued.) The simulation results. (d) The spectrogram of the scrambled speech signal. (e) The time-domain waveform of the descrambled speech signal. (f) The spectrogram of the descrambled speech signal. 


\section{REFERENCES}

[1] V. Lakshmikantham, D. D. Bainov, and P. S. Simeonov, Theory of Impulsive Differential Equations. Singapore: World Scientific, 1989.

[2] E. Ott, C. Grebogi, and J. A. Yorke, "Controlling chaos," Phys. Rev. Lett., vol. 64, pp. 1196-1199, 1990.

[3] G. Chen and X. Dong, "From chaos to order-Perspectives and methodologies in controlling nonlinear dynamical systems," Int. J. Bifurc. Chaos, vol. 3, pp. 1363-1409, 1993.

[4] L. O. Chua, T. Yang, G. Q. Zhong, and C. W. Wu, "Adaptive synchronization of Chua's oscillators," Int. J. Bifurc. Chaos, vol. 6, no. 1, pp. 189-201, 1996.

[5] C. W. Wu, T. Yang, and L. O. Chua, "On adaptive synchronization and control of nonlinear dynamical systems," Int. J. Bifurc. Chaos, vol. 6, no. 3, pp. 455-471, Mar. 1996.

[6] T. Stojanovski, L. Kocarev, and U. Parlitz, "Driving and synchronizing by chaotic impulses," Phys. Rev. E, vol. 43, no. 9, pp. 782-785, Sept. 1996.

[7] K. Pyragas, "Continuous control of chaos by self-controlling feedback," Phys. Lett. A., vol. 170, pp. 421-428, 1992.

[8] J. Schweizer and M. P. Kennedy, "Predictive Poincaré control: A control theory for chaotic systems," Phys. Rev. E, vol. 52, no. 5, pt. A, pp. 4865-4876, Nov. 1995.

[9] E. R. Hunt and G. Johnson, "Keeping chaos at bay," IEEE Spectrum, pp. 32-36, Nov. 1993.

[10] A. M. Samoilenko and N. A. Perestyuk, Impulsive Differential Equations. Singapore: World Scientific, 1995.

[11] L. O. Chua, "Global unfolding of Chua's circuit," IEICE Trans. Fundament., vol. E76-A, no. 5, pp. 704-734, May 1993.

[12] T. Yang, "Recovery of digital signals from chaotic switching," Int. J. Circuit Theory Applicat., vol. 23, no. 6, pp. 611-615, Nov./Dec. 1995

[13] K. M. Short, "Steps toward unmasking secure communications," Int. J. Bifurc. Chaos, vol. 4, pp. 957-977, 1994.

[14] T. Yang, C. W. Wu, and L. O. Chua, "Cryptography based on chaotic systems," IEEE Trans. Circuits Syst. I, vol. 44, pp. 469-472, May 1997.

[15] T. Yang, L.-B. Yang, and C.-M. Yang, "Impulsive synchronization of Lorenz systems," Phys. Lett. A, vol. 226, no. 6, pp. 349-354, Mar. 1997.

[16] R. E. Amritkar and N. Gupte, "Synchronization of chaotic orbits: The effect of a finite time step," Phys. Rev. E (Statistical Physics, Plasmas, Fluids, and Related Interdisciplinary Topics), vol. 47, no. 6, pp. 3889-3895, June 1993.
[17] T. Yang and L. O. Chua, "Impulsive control and synchronization of nonlinear dynamical systems and application to secure communication," Int. J. Bifurc. Chaos, vol. 7, no. 3, pp. 645-664, Mar. 1997.

[18] _ _ "Impulsive control and synchronization of chaotic systems and secure communication," Electron. Res. Lab., College of Eng., Univ. California, Berkeley, Memo. UCB/ERL M97/12, 29 Jan. 29, 1997.

[19] T. Yang, L.-B. Yang, and C.-M. Yang, "Impulsive control of Lorenz system," Physica D, 1997, in press.

[20] T. Yang, C.-M. Yang, and L.-B. Yang, "Control of Rössler system to periodic motions using impulsive control methods," Phys. Lett. A, vol. 232, pp. 356-361, Aug. 4, 1997.

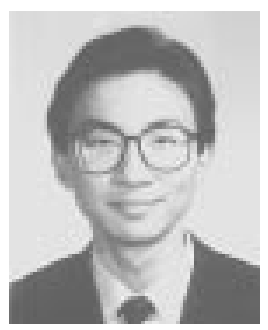

Tao Yang (M'96) was born in Wuhan, China, on January 1, 1970. He received the B.E. and M.E. degrees in electrical engineering from Tongji University, Shanghai, China, in 1990 and 1993 with honors, respectively.

From 1993 to 1994, he was with the Department of Automatic Control Engineering, Shanghai University of Technology, Shanghai. Since 1995, he has been a Specialist in the Nonlinear Electronics Laboratory, Department of Electrical Engineering and Computer Sciences, University of California at Berkeley. His research interests include automatic control, nonlinear dynamic systems, neural networks, fuzzy systems, general brain theory, and supernatural phenomena. He has published more than 40 technical papers in different international journals and different proceedings of international conferences.

Prof. Yang serves as a reviewer of several technical journals including: IEEE TRANSACTIONS ON CIRCUITS AND SySTEMS, International Journal of Bifurcation and Chaos, and SIAM Journal Applied Mathematics. He received the Overseas Professor Chair of E-Zhou University, China, in 1997. He is the founder and the director of E-Zhou Association of Supernatural Phenomena, China.

Leon O. Chua (S'60-M'62-SM'70-F'74), for a biography, see this issue, p. 904 . 